\title{
A MODEL FOR THE NOVA OUTBURST
}

\author{
WILLIAM K. ROSE \\ (Physics Dept., Massachusetts Institute of Technology, Cambridge, Mass., U.S.A.)
}

Models for a $0.75 M_{\odot}$ hydrogen-exhausted star that is approaching the whitedwarf state have been computed. It has been assumed that the star is in a close binary system and therefore can accrete small amounts of hydrogen-rich material $\left(\approx 7 \times 10^{-8}\right.$ $\left.M_{\odot} / \mathrm{yr}\right)$. Hydrogen burning commences when the mass of the hydrogen-rich envelope exceeds about $10^{-4} M_{\odot}$. Eventually the mass accretion causes the non-degenerate hydrogen-burning shell to become thermally unstable, and therefore the nuclear reaction rate increases very dramatically. Pulsational instability is found to develop as a consequence of the very high rate of nuclear-energy generation.

A theory of novae must explain the observed fluxes and mass loss. In addition, it must explain the short rise times as well as the observed dispersion in rise times that characterize the nova outburst. The present model for a nova outburst assumes that the energy released during the period of early decline is energy that has been stored in the form of pulsations. The observations of novae indicate that the amount of energy that is released during the period of early decline is about $3 \times 10^{44}$ ergs. The computed pulsational modes for the models indicate that about $6 \times 10^{44}$ ergs could be stored in the form of pulsational energy for these models.

On the other hand, the calculations suggest that the rise time observed for a nova outburst might be caused by thermal expansion of the outer layers of a thermally unstable star that is also pulsationally unstable. This is true because the relative amplitude of the adiabatic modes in the vicinity of the surface are greatly increased with respect to those for interior regions of the star as a consequence of thermal expansion. This implies that energy in the form of pulsations has been transported closer to the surface.

The present model for a nova outburst assumes that the energy released during the period of slow decline, which may continue for a number of years, is due to the thermal diffusion of photons that were initially emitted during the period of high nuclear energy generation. The maximum surface luminosity that results in the computed models because of the high rate of nuclear-energy generation is approximately 5-6 magnitudes above that for the initial model, and therefore is in agreement with the schematic light curve for a nova outburst due to McLaughlin.

A more complete discussion of these results as well as references to earlier work will be published in a forthcoming issue of the Astrophys. $J$.

Perek (ed.), Highlights of Astronomy, 455. (c) I.A.U. 